\title{
Strong magnetic fields in a nonlocal Polyakov chiral quark model
}

\author{
D. Gómez Dumm ${ }^{1,2, \star}$, M.F. Izzo Villafañe $e^{1,2}$, S. Noguera ${ }^{3}$, V.P. Pagura ${ }^{3}$, and N.N. Scoccola ${ }^{2,4,5}$. \\ ${ }^{1}$ IFLP, CONICET - Departamento de Física, Fac. de Cs. Exactas, Universidad Nacional de La Plata, C.C. \\ 67, (1900) La Plata, Argentina \\ ${ }^{2}$ CONICET, Rivadavia 1917, (1033) Buenos Aires, Argentina \\ ${ }^{3}$ Departamento de Física Teórica and IFIC, Centro Mixto Universidad de Valencia-CSIC, E-46100 Burjassot \\ (Valencia), Spain \\ ${ }^{4}$ Physics Department, Comisión Nacional de Energía Atómica, Av. Libertador 8250, (1429) Buenos Aires, \\ Argentina \\ ${ }^{5}$ Universidad Favaloro, Solís 453, (1078) Buenos Aires, Argentina
}

\begin{abstract}
We study the behavior of strongly interacting matter under an external constant magnetic field in the context of nonlocal chiral quark models that incorporate a coupling to the Polyakov loop. We find that at zero temperature the behavior of the quark condensates shows the expected magnetic catalysis effect, our predictions being in good quantitative agreement with lattice QCD results. On the other hand when the analysis is extended to the case of finite temperature our results show that nonlocal models naturally lead to the Inverse Magnetic Catalysis effect for both the chiral restoration and deconfinement transition temperatures.
\end{abstract}

\section{Introduction}

The study of the behavior of strongly interacting matter under intense external magnetic fields has gained increasing interest in the last few years. In fact, this topic has important applications e.g. in the description of compact objects like magnetars [1], the analysis of heavy ion collisions at very high energies [2] and the exploration of phases at the early Universe [3]. Since these studies require to deal with quantum chromodynamics (QCD) in nonperturbative regimes, present theoretical analyses are based either in the predictions of effective models or in the results obtained through lattice QCD (LQCD) calculations. In particular, the features of QCD phase transitions under external magnetic fields deserve significant interest. Recent reviews on this subject can be found in Refs. [4-6]. In view of the difficulty of theoretical calculations, most works concentrate on the case in which one has a uniform and static external magnetic field $\vec{B}$. At zero temperature and chemical potential, both the results of low-energy effective models of QCD and LQCD calculations indicate that the chiral quark condensates should behave as increasing functions of $B$, which is usually known as "magnetic catalysis". On the contrary, close to the chiral restoration temperature, LQCD calculations carried out with realistic quark masses $[7,8]$ show that light quark-antiquark condensates behave as nonmonotonic functions of the external magnetic field, and this leads to a decrease of the transition temperature when the magnetic field is increased. This effect is known as "inverse magnetic catalysis" (IMC). In addition,

^e-mail: dumm@ fisica.unlp.edu.ar 
LQCD calculations predict an entanglement between the chiral restoration and deconfinement critical temperatures [7]. These findings become a challenge to model calculations. Indeed, most naive effective approaches to low energy QCD (Nambu-Jona-Lasinio model, chiral perturbation theory, MIT bag model, quark-meson models) predict that the chiral transition temperature should grow with $B$, i.e., they do not find IMC. Although many scenarios have been considered in the last few years to account for the IMC [5], the mechanism behind this effect is not yet fully understood.

The aim of this contribution is to present the results of some recent analyses $[9,10]$ of the behavior of strongly interacting matter under a uniform, static magnetic field in the framework of nonlocal chiral quark models. We will show that nonlocal models are able to describe, at the mean field level, not only the IMC effect but also the entanglement between chiral restoration and deconfinement transition temperatures when a coupling to the Polyakov loop is incorporated. The "nonlocal Polyakov-NambuJona-Lasinio" (nlPNJL) models considered here are a sort of nonlocal extensions of the PNJL model that intend to provide a more realistic effective approach to QCD. In fact, nonlocality arises naturally in the context of successful descriptions of low-energy quark dynamics [11, 12], and it has been shown [13] that nonlocal models can lead to a momentum dependence in quark propagators that is consistent with LQCD results. It is also found that in this framework one obtains an adequate description of the properties of light mesons at both zero and finite temperature/density (see e.g. [14] and references therein).

The article is organized as follows. In Sect. II we introduce the formalism to deal with a nonlocal NJL-like model in the presence of the magnetic field at zero temperature. Afterwards we extend this formalism to a finite temperature system, taking into account the coupling to the Polyakov loop. In Sect. III we quote our numerical results, discussing the behavior of the different relevant quantities as functions of the magnetic field and/or temperature. In Sect. IV we present our conclusions.

\section{Theoretical formalism}

Let us start by stating the Euclidean action for our nonlocal NJL-like two-flavor quark model,

$$
S_{E}=\int d^{4} x\left\{\bar{\psi}(x)\left(-i \not \partial+m_{c}\right) \psi(x)-\frac{G}{2} j_{a}(x) j_{a}(x)\right\} .
$$

Here $m_{c}$ is the current quark mass, which is assumed to be equal for $u$ and $d$ quarks. The currents $j_{a}(x)$ are given by

$$
j_{a}(x)=\int d^{4} z \mathcal{G}(z) \bar{\psi}\left(x+\frac{z}{2}\right) \Gamma_{a} \psi\left(x-\frac{z}{2}\right),
$$

where $\Gamma_{a}=\left(\mathbb{1}, i \gamma_{5} \vec{\tau}\right)$, and the function $\mathcal{G}(z)$ is a nonlocal form factor that characterizes the effective interaction. We introduce now in the effective action Eq. (1) a coupling to an external electromagnetic gauge field $\mathcal{A}_{\mu}$. For a local theory this can be done by performing the replacement

$$
\partial_{\mu} \rightarrow D_{\mu} \equiv \partial_{\mu}-i \hat{Q} \mathcal{A}_{\mu}(x),
$$

where $\hat{Q}=\operatorname{diag}\left(q_{u}, q_{d}\right)$, with $q_{u}=2 e / 3, q_{d}=-e / 3$, is the electromagnetic quark charge operator. In the case of the nonlocal model under consideration, the inclusion of gauge interactions implies a change not only in the kinetic terms of the Lagrangian but also in the nonlocal currents in Eq. (2). One has

$$
\psi(x-z / 2) \rightarrow \mathcal{W}(x, x-z / 2) \psi(x-z / 2),
$$

and a related change holds for $\bar{\psi}(x+z / 2)$ [13]. Here the function $\mathcal{W}(s, t)$ is defined by

$$
\mathcal{W}(s, t)=\mathrm{P} \exp \left[-i \int_{s}^{t} d r_{\mu} \hat{Q} \mathcal{A}_{\mu}(r)\right],
$$


where $r$ runs over an arbitrary path connecting $s$ with $t$. As it is usually done, we take it to be a straight line path.

To proceed it is convenient to bosonize the fermionic theory, introducing scalar and pseudoscalar fields $\sigma(x)$ and $\vec{\pi}(x)$ and integrating out the fermion fields. The bosonized action can be written as

$$
S_{\text {bos }}=-\ln \operatorname{det} \mathcal{D}_{x, x^{\prime}}+\frac{1}{2 G} \int d^{4} x[\sigma(x) \sigma(x)+\vec{\pi}(x) \cdot \vec{\pi}(x)],
$$

where

$$
\mathcal{D}_{x, x^{\prime}}=\delta^{(4)}\left(x-x^{\prime}\right)\left(-i \not D+m_{c}\right) \mathcal{G}\left(x-x^{\prime}\right) \gamma_{0} \mathcal{W}(x, \bar{x}) \gamma_{0}\left[\sigma(\bar{x})+i \gamma_{5} \vec{\tau} \cdot \vec{\pi}(\bar{x})\right] \mathcal{W}\left(\bar{x}, x^{\prime}\right),
$$

with $\bar{x}=\left(x+x^{\prime}\right) / 2$. We will consider the case of a constant and homogenous magnetic field orientated along the 3 -axis, choosing the Landau gauge, in which one has $\mathcal{A}_{\mu}=B x_{1} \delta_{\mu 2}$. We assume that the field $\sigma$ has a nontrivial translational invariant mean field value $\bar{\sigma}$, while the mean field values of pseudoscalar fields $\pi_{i}$ are zero. The assumption that $\bar{\sigma}$ is independent of $x$ does not imply that the resulting quark propagator will be translational invariant. It just states that the deviations from translational invariance driven by the magnetic field are not affected by the dynamics of the theory. In this way, within the mean field approximation (MFA) we get

$$
\mathcal{D}_{x, x^{\prime}}^{\mathrm{MFA}}=\operatorname{diag}\left(\mathcal{D}_{x, x^{\prime}}^{\mathrm{MFA}, u} \mathcal{D}_{x, x^{\prime}}^{\mathrm{MFA}, d}\right),
$$

where

$$
\mathcal{D}_{x, x^{\prime}}^{\mathrm{MFA}, f}=\delta^{(4)}\left(x-x^{\prime}\right)\left(-i \not \partial-q_{f} B x_{1} \gamma_{2}+m_{c}\right)+\bar{\sigma} \mathcal{G}\left(x-x^{\prime}\right) \exp \left[i\left(q_{f} B / 2\right)\left(x_{1}+x_{1}^{\prime}\right)\left(x_{2}-x_{2}^{\prime}\right)\right] .
$$

To deal with this operator it is convenient to introduce its Ritus transform $\mathcal{D}_{\bar{p}, \bar{p}^{\prime}}^{\mathrm{MFA}, f}$, defined by

$$
\mathcal{D}_{\bar{p}, \bar{p}^{\prime}}^{\mathrm{MFA}, f}=\int d^{4} x d^{4} x^{\prime} \overline{\mathbb{E}}_{\bar{p}}(x) \mathcal{D}_{x, x^{\prime}}^{\mathrm{MFA}, f} \mathbb{E}_{\bar{p}^{\prime}}\left(x^{\prime}\right),
$$

where $\mathbb{E}_{\bar{p}}(x)$ and $\overline{\mathbb{E}}_{\bar{p}}(x)$, with $\bar{p}=\left(k, p_{2}, p_{3}, p_{4}\right)$, are Ritus functions [15]. The index $k$ is an integer that will label the Landau energy levels. Using the properties of Ritus functions, and after some calculation, we obtain

$$
\mathcal{D}_{\bar{p}, \bar{p}^{\prime}}^{\mathrm{MFA}, f}=(2 \pi)^{4} \delta_{k k^{\prime}} \delta\left(p_{2}-p_{2}^{\prime}\right) \delta\left(p_{3}-p_{3}^{\prime}\right) \delta\left(p_{4}-p_{4}^{\prime}\right) \mathcal{D}_{k, p_{\|}}^{f},
$$

where

$$
\mathcal{D}_{k, p_{\|}}^{f}=P_{k, s_{f}}\left(-s_{f} \sqrt{2 k\left|q_{f} B\right|} \gamma_{2}+p_{\|} \cdot \gamma_{\|}\right)+\sum_{\lambda= \pm} M_{k, p_{\|}}^{\lambda, f} \Delta^{\lambda}
$$

Here we have introduced the definitions $s_{f}=\operatorname{sign}\left(q_{f} B\right), p_{\|}=\left(p_{3}, p_{4}\right), \gamma_{\|}=\left(\gamma_{3}, \gamma_{4}\right), \Delta^{+}=$ $\operatorname{diag}(1,0,1,0), \Delta^{-}=\operatorname{diag}(0,1,0,1)$ and $P_{k, \pm 1}=\left(1-\delta_{k 0}\right) \mathcal{I}+\delta_{k 0} \Delta^{ \pm}$. In addition, we denote

$$
M_{k, p_{\|}}^{\lambda, f}=\frac{4 \pi}{\left|q_{f} B\right|}(-1)^{k_{\lambda}} \int \frac{d^{2} p_{\perp}}{(2 \pi)^{2}}\left(m_{c}+\sigma g\left(p_{\perp}^{2}+p_{\|}^{2}\right)\right) \exp \left(-p_{\perp}^{2} /\left|q_{f} B\right|\right) L_{k_{\lambda}}\left(2 p_{\perp}^{2} /\left|q_{f} B\right|\right) .
$$

where we have used the definitions $k_{ \pm}=k-1 / 2 \pm s_{f} / 2$ and $p_{\perp}=\left(p_{1}, p_{2}\right)$, while $g\left(p^{2}\right)$ is the Fourier transform of $\mathcal{G}(x)$ and $L_{m}(x)$ are Laguerre polynomials, with the usual convention $L_{-1}(x)=0$.

Using the fact that $\mathcal{D}^{\mathrm{MFA}, f}$ is diagonal in Ritus space the corresponding contribution to the MFA action can be readily calculated. We obtain

$$
\frac{S_{\mathrm{bos}}^{\mathrm{MFA}}}{V^{(4)}}=\frac{\bar{\sigma}^{2}}{2 G}-N_{c} \sum_{f=u, d} \frac{\left|q_{f} B\right|}{2 \pi} \int \frac{d^{2} p_{\|}}{(2 \pi)^{2}}\left[\ln \left(p_{\|}^{2}+M_{0, p_{\|}}^{\lambda_{f}, f^{2}}\right)+\sum_{k=1}^{\infty} \ln \Delta_{k, p_{\|}}^{f}\right],
$$


where $\lambda_{f}=+(-)$ for $s_{f}=+1(-1)$, and $\Delta_{k, p_{\|}}^{f}$ is defined by

$$
\Delta_{k, p_{\|}}^{f}=\left(2 k\left|q_{f} B\right|+p_{\|}^{2}+M_{k, p_{\|}}^{+, f} M_{k, p_{\|}}^{-, f}\right)^{2}+p_{\|}^{2}\left(M_{k, p_{\|}}^{+, f}-M_{k, p_{\|}}^{-, f}\right)^{2}
$$

Here it is seen that the functions $M_{k, p_{\|}}^{ \pm, f}$ play the role of constituent quark masses in the presence of the external magnetic field.

We extend now the analysis to a system at finite temperature. This is done by using the standard Matsubara formalism. To account for confinement effects, we also include the coupling of fermions to the Polyakov loop (PL), assuming that quarks move on a constant color background field $\phi=$ ig $\delta_{\mu 0} G_{a}^{\mu} \lambda^{a} / 2$, where $G_{a}^{\mu}$ are the SU(3) color gauge fields. We work in the so-called Polyakov gauge, in which the matrix $\phi$ is given a diagonal representation $\phi=\phi_{3} \lambda_{3}+\phi_{8} \lambda_{8}$, taking the traced Polyakov loop $\Phi=\frac{1}{3} \operatorname{Tr} \exp (i \phi / T)$ as an order parameter of the confinement/deconfinement transition. Since owing to the charge conjugation properties of the QCD Lagrangian - the mean field traced Polyakov loop is expected to be a real quantity, and $\phi_{3}$ and $\phi_{8}$ are assumed to be real valued [16], one has $\phi_{8}=0$, $\Phi=\left[1+2 \cos \left(\phi_{3} / T\right)\right] / 3$. Finally, we include in the Lagrangian a Polyakov-loop potential $\mathcal{U}(\Phi, T)$, which accounts for effective gauge field self-interactions. The resulting scheme is usually denoted as nonlocal Polyakov-Nambu-Jona-Lasinio (nlPNJL) model [17, 18].

Concerning the PL potential, its functional form is usually based on properties of pure gauge QCD. In this work we will mostly focus on a potential given by a polynomial function based on a Ginzburg-Landau ansatz [19], namely

$$
\frac{\mathcal{U}_{\mathrm{poly}}(\Phi, T)}{T^{4}}=-\frac{b_{2}(T)}{2} \Phi^{2}-\frac{b_{3}}{3} \Phi^{3}+\frac{b_{4}}{4} \Phi^{4}, \quad b_{2}(T)=\sum_{n=0}^{3} a_{n}\left(\frac{T_{0}}{T}\right)^{n}
$$

The parameters $a_{i}$ and $b_{i}$ can be fitted to pure gauge lattice QCD results imposing the presence of a first-order phase transition at $T_{0}$, which is a further parameter of the model. In the absence of dynamical quarks, from lattice calculations one expects a deconfinement temperature $T_{0}=270 \mathrm{MeV}$. However, it has been argued that in the presence of light dynamical quarks this temperature scale should be considerably reduced. Following Ref. [20] we use here $T_{0}=210 \mathrm{MeV}$, taking the values of $a_{i}$ and $b_{i}$ from Ref. [19].

In this way, the grand canonical thermodynamic potential of the system under the external magnetic field is found to be given by

$$
\Omega_{B, T}^{\mathrm{MFA}}=\frac{\bar{\sigma}^{2}}{2 G}-T \sum_{n=-\infty}^{\infty} \sum_{c, f} \frac{\left|q_{f} B\right|}{2 \pi} \int \frac{d p_{3}}{2 \pi}\left[\ln \left(p_{\|_{n c}}^{2}+M_{0, p_{\| n c}}^{\lambda_{f},{ }^{2}}\right)+\sum_{k=1}^{\infty} \ln \left(\Delta_{k, p_{\| n c}}^{f}\right)\right]+\mathcal{U}(\Phi, T)
$$

where we have defined $p_{\|_{n c}}=\left(p_{3},(2 n+1) \pi T+\phi_{c}\right)$. The sums over color and flavor indices run over $c=r, g, b$ and $f=u, d$, respectively, while the color background fields are $\phi_{r}=-\phi_{g}=\phi_{3}, \phi_{b}=0$. As usual in nonlocal models, it is seen that $\Omega^{\mathrm{MFA}}$ turns out to be divergent, thus it has to be regularized. We take here the usual subtraction prescription

$$
\Omega_{B, T}^{\mathrm{MFA}, \text { reg }}=\Omega_{B, T}^{\mathrm{MFA}}-\Omega_{B, T}^{\mathrm{free}}+\Omega_{B, T}^{\mathrm{free}, \mathrm{reg}},
$$

where the "free" contribution stands for the potential obtained in absence of the strong current-current coupling (i.e. setting $\bar{\sigma}=0$ ), but keeping the interaction with the magnetic field and the PL. For this 
"free" piece the Matsubara sum can be performed analytically, leading to

$$
\begin{aligned}
\Omega_{B, T}^{\text {free,reg }}= & -\frac{N_{c}}{2 \pi^{2}} \sum_{f}\left(q_{f} B\right)^{2}\left[\zeta^{\prime}\left(-1, x_{f}\right)+\frac{x_{f}^{2}}{4}-\frac{1}{2}\left(x_{f}^{2}-x_{f}\right) \ln x_{f}\right]- \\
& T \sum_{f, c} \frac{\left|q_{f} B\right|}{\pi} \sum_{k=0}^{\infty} \alpha_{k} \int \frac{d p}{2 \pi} \ln \left\{1+\exp \left[-\left(\epsilon_{k p}^{f}+i \phi_{c}\right) / T\right]\right\} .
\end{aligned}
$$

Here we have defined $x_{f}=m_{c}^{2} /\left(2\left|q_{f} B\right|\right), \alpha_{k}=2-\delta_{k 0}, \epsilon_{k p}^{f}=\left(2 k\left|q_{f} B\right|+p^{2}+m_{c}^{2}\right)^{1 / 2}$, while $\zeta^{\prime}\left(-1, x_{f}\right)=$ $d \zeta\left(z, x_{f}\right) /\left.d z\right|_{z=-1}$, where $\zeta\left(z, x_{f}\right)$ is the Hurwitz zeta function. Owing to the presence of the background field, one has now a set of two coupled "gap equations"

$$
\frac{\partial \Omega_{B, T}^{\mathrm{MFA}, \mathrm{reg}}}{\partial \bar{\sigma}}=0, \quad \frac{\partial \Omega_{B, T}^{\mathrm{MFA}, \mathrm{reg}}}{\partial \Phi}=0 .
$$

Given $\Omega_{B, T}^{\mathrm{MFA}, \mathrm{reg}}$, the magnetic field dependent quark condensates $\left\langle\bar{q}_{f} q_{f}\right\rangle$ can be calculated by taking the derivatives with respect to the corresponding current quark masses. To make contact with the LQCD results quoted in Ref. [8] we define the quantities

$$
\Sigma_{B, T}^{f}=-\frac{2 m_{c}}{S^{4}}\left[\left\langle\bar{q}_{f} q_{f}\right\rangle_{B, T}^{\mathrm{reg}}-\langle\bar{q} q\rangle_{0,0}^{\mathrm{reg}}\right]+1
$$

where $S=(135 \times 86)^{1 / 2} \mathrm{MeV}$. The subindex $f$ can be omitted for $B=0$, owing to isospin symmetry. We also introduce the definitions $\Delta \Sigma_{B, T}^{f}=\Sigma_{B, T}^{f}-\Sigma_{0, T}^{f}, \bar{\Sigma}_{B, T}=\left(\Sigma_{B, T}^{u}+\Sigma_{B, T}^{d}\right) / 2$ and $\Delta \bar{\Sigma}_{B, T}=\left(\Delta \Sigma_{B, T}^{u}+\right.$ $\left.\Delta \Sigma_{B, T}^{d}\right) / 2$, which correspond to the subtracted normalized flavor condensate, the normalized flavor average condensate and the subtracted normalized flavor average condensate, respectively.

\section{Numerical results}

To obtain numerical predictions for the behavior of the above defined quantities as functions of the temperature and the external magnetic field, it is necessary to specify the particular shape of the nonlocal form factor $g\left(p^{2}\right)$. We consider here two often-used forms, namely a Gaussian function and a "5-Lorentzian" function, defined by

$$
g\left(p^{2}\right)=\exp \left(-p^{2} / \Lambda^{2}\right), \quad g\left(p^{2}\right)=\frac{1}{1+\left(p^{2} / \Lambda^{2}\right)^{5}},
$$

respectively. Notice that in these form factors we introduce an energy scale $\Lambda$, which acts as an effective momentum cut-off. This has to be taken as an additional parameter of the model. Given the nonlocal form factor, one has to determine the values of the parameters $m_{c}, G$ and $\Lambda$. We consider different parameter sets, obtained by requiring that the model leads to the empirical values of the pion mass and decay constant, as well as some phenomenologically acceptable value of the quark condensate at $B=0$ and $T=0$. We take in particular $\left(-\langle\bar{q} q\rangle_{0,0}^{\mathrm{reg}}\right)^{1 / 3}=220,230$ and $240 \mathrm{MeV}$. The corresponding parameter sets for the Gaussian and 5-Lorentzian form factors can be found e.g. in Ref. [10].

Let us start by discussing our results for zero temperature. In Fig. 1 we show the predictions of our model for $\Delta \bar{\Sigma}_{B, 0}$ (left panel) and $\Sigma_{B, 0}^{u}-\Sigma_{B, 0}^{d}$ (right panel) as functions of $e B$. LQCD data from Ref. [8] are also displayed in both cases for comparison. Solid, dashed and dotted curves correspond to $\left(-\left\langle\bar{q}_{f} q_{f}\right\rangle_{0,0}^{\mathrm{reg}}\right)^{1 / 3}=220,230$ and $240 \mathrm{MeV}$, respectively, for the case of a Gaussian form factor. It can 
be seen that the predictions for $\Delta \bar{\Sigma}_{B, 0}$ are very similar for all parametrizations considered, and show a very good agreement with LQCD results. In the case of $\Sigma_{B, 0}^{u}-\Sigma_{B, 0}^{d}$, although the overall agreement with LQCD calculations is still good, we find some dependence on the parameterization. For the case of the 5-Lorentzian form factor our results turn out to be qualitatively similar to those in Fig. 1.
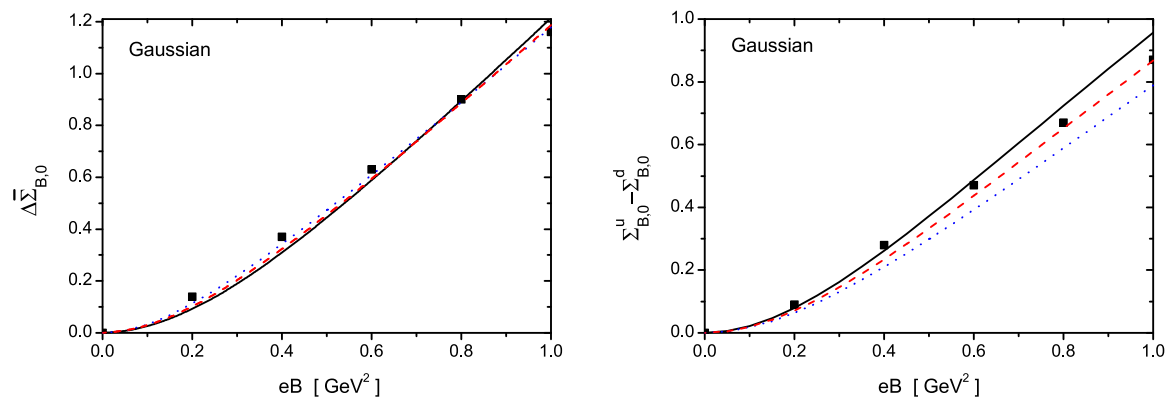

Figure 1. Normalized condensates as functions of the magnetic field at $T=0$, for the case of a Gaussian form factor. Left panel: subtracted flavor average; right panel: flavor difference [see Eq. (21) and the text below]. Solid (black), dashed (red) and dotted (blue) curves correspond to parameterizations leading to $\left(-\langle\bar{q} q\rangle_{0,0}^{\text {reg }}\right)^{1 / 3}=220,230$ and $240 \mathrm{MeV}$, respectively. Full square symbols indicate LQCD results taken from Ref. [8].

We turn now to our numerical results for a system at finite temperature. In the upper panels of Fig. 2 we show the behavior of the averaged chiral condensate $\bar{\Sigma}_{B, T}$ and the traced Polyakov loop $\Phi$ as functions of the temperature, for three representative values of the external magnetic field $B$, namely $B=0,0.6$ and $1 \mathrm{GeV}^{2}$. The curves correspond to parameter sets leading to $\left(-\langle\bar{q} q\rangle_{0,0}^{\mathrm{reg}}\right)^{1 / 3}=230 \mathrm{MeV}$. Given a value of $B$, it is seen from the figure that for the cases of both Gaussian and 5-Lorentzian form factors the chiral restoration and deconfinement transitions proceed as smooth crossovers, at approximately the same critical temperatures. For definiteness we take these temperatures from the maxima of the chiral and PL susceptibilities, which we define as the derivatives $\chi_{\mathrm{ch}}=-\partial\left[\left(\langle\bar{u} u\rangle_{B, T}^{\mathrm{reg}}+\right.\right.$ $\left.\left.\langle\bar{d} d\rangle_{B, T}^{\mathrm{reg}}\right) / 2\right] / \partial T$ and $\chi_{\Phi}=\partial \Phi / \partial T$, respectively. Our results for the behavior of the susceptibilities as functions of the temperature, for $B=0,0.6$ and $1 \mathrm{GeV}^{2}$, are shown in the lower panels of Fig. 2.

Table 1. Critical temperatures for $B=0$ and various parametrizations.

\begin{tabular}{l|ccc|ccc} 
& \multicolumn{3}{|c}{ Gaussian } & \multicolumn{3}{c}{ 5-Lorentzian } \\
$\left(-\langle q \bar{q}\rangle_{0,0}^{\mathrm{reg}}\right)^{1 / 3}(\mathrm{MeV})$ & 220 & 230 & 240 & 220 & 230 & 240 \\
\hline Chiral $T_{c}(\mathrm{MeV})$ & 182.1 & 179.1 & 177.4 & 177.0 & 177.0 & 177.8 \\
Deconfinement $T_{c}(\mathrm{MeV})$ & 182.1 & 178.0 & 175.8 & 174.8 & 174.7 & 175.5
\end{tabular}

The chiral restoration and deconfinement critical temperatures obtained in absence of external magnetic field for different parametrizations are quoted in Table 1. It is seen that in all cases the splitting between both critical temperatures is below $5 \mathrm{MeV}$, which is consistent with the results obtained in lattice QCD. From Table 1 it is also seen that the values of critical temperatures do not vary significantly with the parametrization (recalling that in all cases the parameters have been fixed to reproduce the empirical values of the pion mass and decay constant). On the other hand, the critical temperatures in Table 1 are found to be somewhat higher than those obtained from LQCD, which lie 

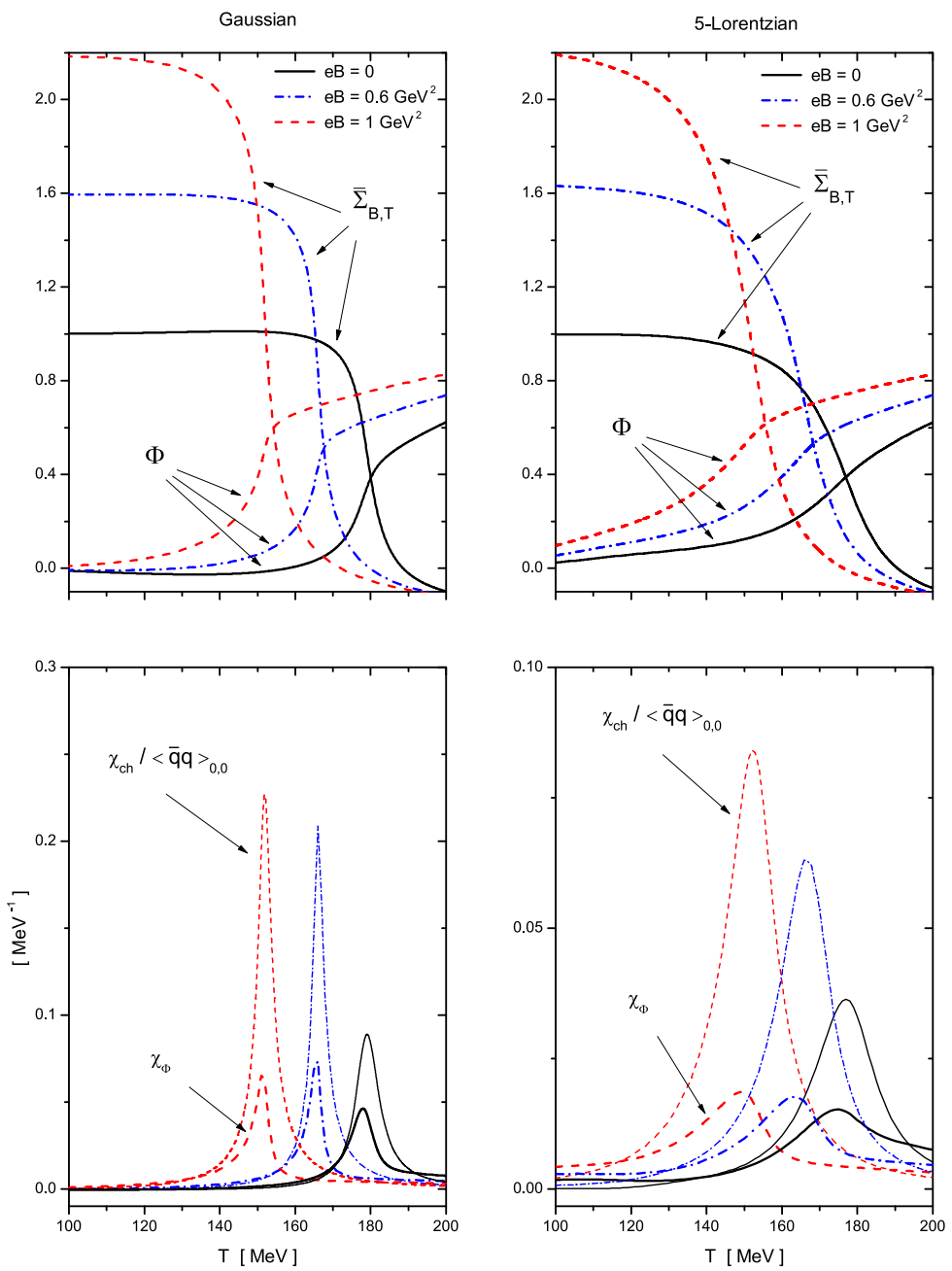

Figure 2. Upper panels: normalized flavor average condensate and traced Polyakov loop as functions of the temperature, for three representative values of $e B$. Lower panels: behavior of the corresponding chiral and PL susceptibilities as functions of the temperature.

around $160 \mathrm{MeV}[21,22]$. It is worth noticing that in absence of the interaction with the Polyakov loop the values of $T_{c}$ drop down to about $130 \mathrm{MeV}$ [9].

Let us discuss the effect of the magnetic field on the phase transition features. From Fig. 2 it is seen that the splitting between the chiral restoration and deconfinement critical temperatures remains very small in the presence of the external field (in fact, a detailed analysis shows that the splitting gets reduced for larger values of $e B$ ). In addition, it is seen that the nonlocal NJL models show inverse magnetic catalysis. Indeed, contrary to what happens e.g. in the standard local NJL model [4-6], in our models the chiral restoration critical temperature becomes lower as the external magnetic field is increased. This is related with the fact that the condensates do not show in general a monotonic 
increase with $B$ for a fixed value of the temperature. The situation is illustrated in Fig. 3, where we show the behavior of the averaged difference $\Delta \bar{\Sigma}_{B, T}$ as a function of $e B$, for $T=0$ and for values of the temperature in the critical region. The curves correspond to models with Gaussian (left) and Lorentzian (right) form factors, with $\left(-\langle\bar{q} q\rangle_{0,0}^{\text {reg }}\right)^{1 / 3}=230 \mathrm{MeV}$. For these parametrizations the critical temperatures for $B=0$ are slightly below $180 \mathrm{MeV}$ (see Table 1). While for $T=0$ the value of $\Delta \bar{\Sigma}_{B, 0}$ shows a monotonic growth with the external magnetic field, it is seen that when the temperatures get closer to the critical values the curves have a maximum and then start to decrease for increasing $B$. This is the typical behavior associated to IMC and observed from lattice QCD results, see e.g. Fig. 2 of Ref. [8]. Qualitatively similar results are found for the other parametrizations in Table I. Finally, in Fig. 4 we plot our results for the chiral restoration critical temperatures $T_{c}(B)$, normalized to the corresponding values at vanishing external magnetic field. The figure includes the curves for nonlocal NJL models with Gaussian (left) and 5-Lorentzian (right) form factors and different parameter sets (see caption), and the gray bands in both panels show the results obtained in LQCD, taken from Ref. [8]. From the figure it is clearly seen that the inverse magnetic catalysis effect is observed for all considered parametrizations. In addition, for a given form factor the effect is found to be stronger for parameter sets leading to a lower absolute value of the chiral quark condensates. As a general conclusion, it can be stated that the behavior of the critical temperatures with the external magnetic field is compatible with LQCD results, for phenomenologically adequate values of the chiral condensate.
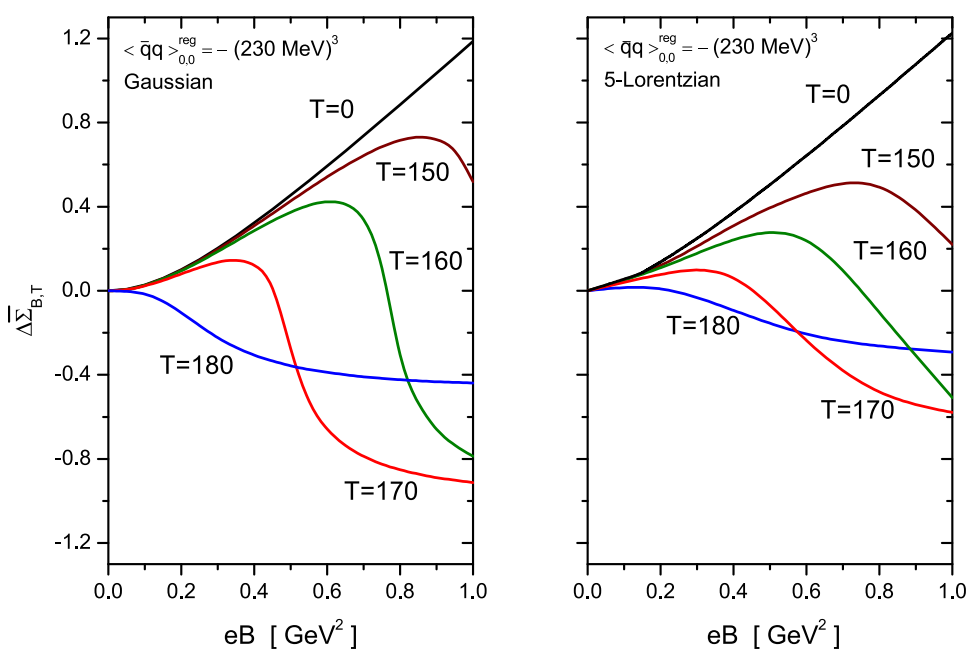

Figure 3. Subtracted normalized flavor average condensate as a function of $e B$ for different representative temperatures. Left and right panels correspond to Gaussian and 5-Lorentzian form factors, respectively, with $\left(-\langle\bar{q} q\rangle_{0,0}^{\mathrm{reg}}\right)^{1 / 3}=230 \mathrm{MeV}$. Temperature values are given in $\mathrm{MeV}$.

\section{Summary \& conclusions}

We have studied the behavior of strongly interacting matter under a uniform static external magnetic field in the context of a nonlocal chiral quark model. In this approach, which can be viewed as 

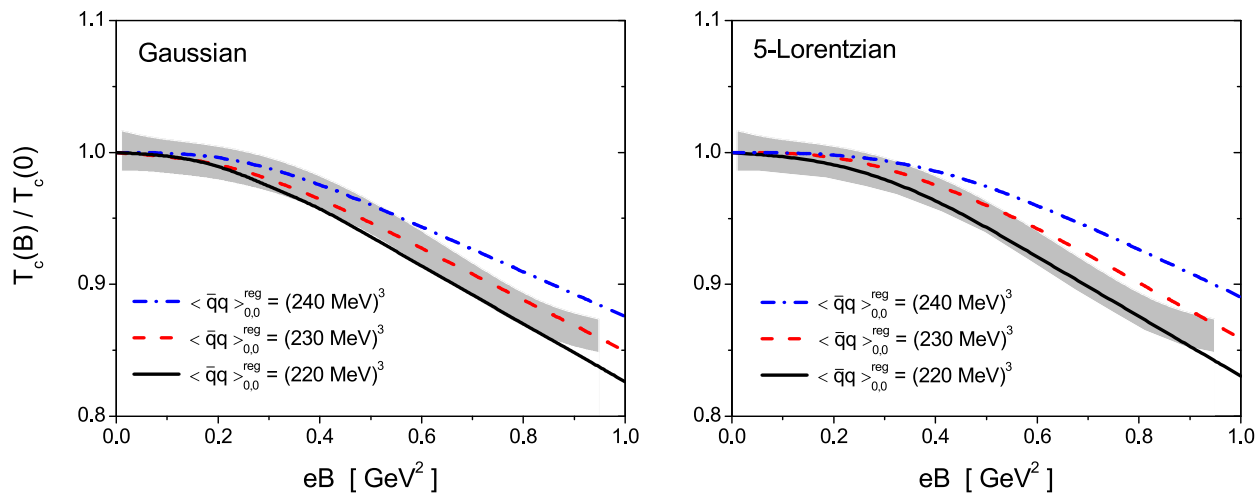

Figure 4. Normalized critical temperatures as functions of $e B$ for various model parametrizations. For comparison, LQCD results of Ref. [8] are indicated by the gray band. Left and right panels correspond to Gaussian and 5-Lorentzian form factors, respectively.

an extension of the Polyakov-Nambu-Jona-Lasinio model, the effective couplings between quarkantiquark currents include nonlocal form factors that regularize ultraviolet divergences in quark loop integrals and lead to a momentum-dependent effective mass in quark propagators. We have worked out the formalism introducing Ritus transforms of Dirac fields, which allow to obtain closed analytical expressions for the gap equations, the chiral quark condensate and the quark propagator.

We have considered the case of Gaussian and Lorentzian form factors, choosing some sets of model parameters that allow to reproduce the empirical values of the pion mass and decay constants. At zero temperature, with these parameterizations we have calculated the behavior of the subtracted flavor average condensate $\Delta \bar{\Sigma}_{B, 0}$ and the normalized condensate difference $\Sigma_{B, 0}^{u}-\Sigma_{B, 0}^{d}$ as functions of the external magnetic field $B$. Our results show the expected effect of magnetic catalysis (condensates behave as growing functions of $B$ ), the curves being in quantitative agreement with lattice QCD calculations with slight dependence on the parametrization.

Finally we have extended the calculations to finite temperature systems, including the couplings of fermions to the Polyakov loop. We have defined chiral and PL susceptibilities in order to study the chiral restoration and deconfinement transitions, which turn out to proceed as smooth crossovers for the considered polynomial PL potential. From our numerical calculations, on one hand it is seen that, for all considered values of $B$, both transitions take place at approximately the same temperature, in agreement with LQCD predictions. On the other hand, it is found that for temperatures close to the transition region $\Delta \bar{\Sigma}_{B, T}$ becomes a nonmonotonic funtion of $B$, which eventually leads to the phenomenon of inverse magnetic catalysis, i.e., a decrease of the critical temperature when the magnetic field gets increased. This feature is also in qualitative agreement with LQCD expectations. Moreover, for some parameterizations we find a remarkably good quantitative agreement with the results from LQCD calculations for the behavior of the normalized critical temperatures with $B$ (see Fig. 4). The values of the critical temperature at $T=0$, which show some dependence on the parameterization, lie also within the range estimated by LQCD results.

It is interesting to compare the nonlocal models with approaches in which IMC is obtained by considering some dependence of the effective couplings on $B$ and/or $T[23,24]$. The naturalness of the IMC behavior in our framework can be understood by noticing that for a given Landau level the associated nonlocal form factor turns out to be a function of the external magnetic field, according to 
the convolution in Eq. (13). Since the form factors can be identified with some gluon-mediated effective interaction, the dependence on the magnetic field can be seen as originated by the backreaction of the quarks on the gluon fields.

\section{Acknowledgements}

This work has been supported in part by CONICET and ANPCyT (Argentina), grants PIP14-492, PIP12-449, and PICT14-03-0492, by UNLP (Argentina), Project X718, by the Mineco (Spain), contract FPA2013-47443-C2-1-P, FPA2016-77177-C2-1-P, by Centro de Excelencia Severo Ochoa Programme, grant SEV-2014-0398, and by Generalitat Valenciana (Spain), grant PrometeoII/2014/066.

\section{References}

[1] R. C. Duncan and C. Thompson, Astrophys. J. 392, L9 (1992); C. Kouveliotou et al., Nature 393, 235 (1998).

[2] D. E. Kharzeev, L. D. McLerran and H. J. Warringa, Nucl. Phys. A 803, 227 (2008); V. Skokov, A. Y. Illarionov, and V. Toneev, Int. J. Mod. Phys. A 24, 5925 (2009); V. Voronyuk et al., Phys. Rev. C 83, 054911 (2011).

[3] T. Vachaspati, Phys. Lett. B265, 258 (1991); K. Enqvist and P. Olesen, Phys. Lett. B319, 178 (1993).

[4] D. E. Kharzeev, K. Landsteiner, A. Schmitt and H. U. Yee, Lect. Notes Phys. 871, 1 (2013).

[5] J. O. Andersen, W. R. Naylor and A. Tranberg, Rev. Mod. Phys. 88, 025001 (2016).

[6] V. A. Miransky and I. A. Shovkovy, Phys. Rept. 576, 1 (2015).

[7] G. S. Bali et al., JHEP 1202, 044 (2012).

[8] G. S. Bali et al., Phys. Rev. D 86, 071502 (2012).

[9] V. P. Pagura, D. Gomez Dumm, S. Noguera and N. N. Scoccola, Phys. Rev. D 95, 034013 (2017).

[10] D. Gomez Dumm, M. F. I. Villafañe, S. Noguera, V. P. Pagura and N. N. Scoccola, arXiv:1709.04742 [hep-ph].

[11] T. Schafer and E. V. Shuryak, Rev. Mod. Phys. 70, 323 (1998).

[12] C. D. Roberts and A. G. Williams, Prog. Part. Nucl. Phys. 33, 477 (1994); C. D. Roberts and S. M. Schmidt, Prog. Part. Nucl. Phys. 45, S1 (2000).

[13] S. Noguera and N. N. Scoccola, Phys. Rev. D 78, 114002 (2008).

[14] G. A. Contrera, D. G. Dumm and N. N. Scoccola, Phys. Rev. D 81, 054005 (2010).

[15] V. I. Ritus, Sov. Phys. JETP 48, 788 (1978).

[16] S. Roessner, C. Ratti and W. Weise, Phys. Rev. D 75, 034007 (2007).

[17] G. A. Contrera, D. Gomez Dumm and N. N. Scoccola, Phys. Lett. B 661, 113 (2008).

[18] T. Hell, S. Roessner, M. Cristoforetti and W. Weise, Phys. Rev. D 79, 014022 (2009).

[19] C. Ratti, M. A. Thaler and W. Weise, Phys. Rev. D 73, 014019 (2006).

[20] B. -J. Schaefer, J. M. Pawlowski and J. Wambach, Phys. Rev. D 76, 074023 (2007); B. -J. Schaefer, M. Wagner and J. Wambach, Phys. Rev. D 81, 074013 (2010).

[21] Y. Aoki et al., JHEP 0906, 088 (2009); S. Borsanyi et al., JHEP 1009, 073 (2010).

[22] A. Bazavov et al., Phys. Rev. D 85, 054503 (2012).

[23] A. Ayala, M. Loewe, A. J. Mizher and R. Zamora, Phys. Rev. D 90, 036001 (2014).

[24] R. L. S. Farias, K. P. Gomes, G. I. Krein and M. B. Pinto, Phys. Rev. C 90, 025203 (2014). 